\title{
Comercio electrónico en la comercialización de dulces artesanales
}

Electronic commerce in the commercialization of artisan sweets

José Luis Merino Murillo, $\mathrm{MSc}^{(1)}$

Dolores Monserrate Yoza Castro, Ing ${ }^{(2)}$

Jenny Parrales Reyes, $\mathrm{MSc}^{(3)}$

(1), (2), (3) Carrera de comercio exterior. Universidad estatal del sur de Manabí. Ecuador

Contacto: jose.merino@unesum.edu.ec, joselu_merino28@hotmail.com

Receptado: 05/02/2018 Aceptado: 16/04/2018

\section{Resumen}

El comercio electrónico es una herramienta tecnológica que actualmente se utiliza para establecer vínculos de ventas directas entre el ofertante y el consumidor, brindando facilidades en su comercialización. La investigación tuvo como objetivo analizar la actividad comercial de los dulces artesanales derivados de la caña de azúcar que produce y comercializa la microempresa Buena Fe y establecer el comercio electrónico como un instrumento para incrementar la comercialización de los mismos. El problema que motivó el estudio se centra en los bajos niveles de comercialización. En el proceso investigativo se utilizó como métodos teóricos, el descriptivo, analítico, sintético y bibliográfico que ayudaron a establecer las bases teóricas de la investigación. La técnica que se empleó fue la encuesta, que fue dirigida a los productores de la microempresa Buena Fe para conocer sus necesidades, opiniones y con aquello llegar a un levantamiento de datos para su posterior análisis e interpretación. Con los resultados obtenidos se pretende mostrar a los microempresarios que existe una herramienta informática como lo es el comercio electrónico que podrían utilizar para dar a conocer sus productos, conseguir nuevos mercados y vender sus productos.

Palabras claves: Alfeñique, comercio electrónico, comercialización, microempresa, dulces artesanales 


\title{
Electronic commerce in the marketing of artisanal sweets
}

\begin{abstract}
E-commerce is a technological tool that is currently used to establish direct sales links between the provider and the consumer, providing facilities for marketing. The objective of this article is to analyze the commercial activity of the handcrafted sweets derived from the sugar cane that produces and commercializes the "Buena Fe" microenterprise good faith and to establish the electronic commerce as an instrument to increase the commercialization of the same ones. The main problem focuses on low levels of marketing, in the investigative process was used as theoretical, descriptive, analytical, synthetic and bibliographical methods that helped to stablish the theoretical bases of research. The technique that was used was the survey, which was directed to producers of the "Buena Fe" microenterprise to know their needs, opinions and with that to reach a data collection for further analysis and interpretation. With the results it is intended to show the micro-entrepreneurs that there is a computer tool like the electronic commerce that they could use to make known their products, to obtain new markets and to sell their products.
\end{abstract}

Keywords: alfeñique, trading, marketing, microenterprise, handcrafted sweets

\section{Introducción}

E-commerce o Comercio Electrónico consiste en la distribución, venta, compra, marketing y suministro de información de productos o servicios a través de Internet. (Redempresariosvisa, 2016). La cantidad de comercio electrónico ha crecido en gran manera debido a Internet. Una gran variedad de comercio se realiza de esta manera, estimulando la creación y utilización de innovaciones como la transferencia de fondos electrónicos, la administración de cadenas de suministro, el marketing en Internet. (Observatoriotic, 2015)

Sin una estrategia de marketing de comercio electrónico, un negocio en línea podría fallar en conjunto con las miles de compañías que venden productos similares a precios similares, es decir con la aplicación de acciones tales como un diseño atractivo de la página, facilidad para navegar en el sitio, catálogo de productos, precio competitivo, ofertas, una buena gestión de stock, el pago por click, seguridad informática y entre otras puede hacer que los clientes no solo sean observadores de la tienda virtual sino que realicen compras, sirvan de nexo con otros clientes y se conviertan en consumidores repetitivos. (Posgradoenmarketing, 2014) 
(Guerreo \& Vera, 2016) Como perspectiva sobre las Microempresas actuales indican que pueden beneficiarse de herramientas como el internet, diseño de páginas virtuales, correo electrónico que con una base de clientes, pudieran abarcar un nicho de mercado, y se podrían utilizar en muchos aspectos como publicidad, atención al cliente, generar ventas en línea, o realizar controles de calidad postventa, todo esto permitiría a las Microempresas, elaborar reportes y hacer la debida retroalimentación, para ofrecer un servicio de calidad. Hay que notar que los clientes actualmente han evolucionado, y cada uno tiene un comportamiento específico, estos clientes esperan que el producto adquirido brinde un valor añadido.

Existen varios tipos de relación comercial en los que se puede aplicar el comercio electrónico, que va de acuerdo a la tipo de empresa entre estas se encuentran: B2C (Business-toConsumer): Empresas que venden al público en general; B2B (Business-toBusiness): Empresas haciendo negocios entre ellas; B2G (Business-to-Government): Empresas que venden a instituciones de gobierno; y, C2C (Consumer-to-Consumer): Plataforma a partir de la cual los consumidores compran y venden entre ellos. (Redempresariosvisa, 2016)

El comercio electrónico realizado entre empresas es llamado en inglés business-to-business o B2B. El B2B puede estar abierto a cualquiera que esté interesado (como el intercambio de mercancías o materias primas entre un negocio y otro), o estar limitado a participantes específicos pre-calificados (mercado electrónico privado). Algunas de sus ventajas son: mejoras en la distribución. (Millan, 2016)

Una de las principales desventajas el comercio electrónico en todo el mundo es la desconfianza. Existe desconfianza tanto de los consumidores como de la empresa. "La desconfianza por el tema de la seguridad en las transacciones y los medios de pagos electrónicos" es uno de los principales problemas del comercio electrónico, por tal razón una tienda electrónica debe de contar con un certificado digital el cual va a permitir que las transacciones entre el navegador y el servidor sean seguras y que se pueda comprobar que el negocio es real. (Landivar , 2012)

(Chillogalli, 2015) En su proyecto de tesis menciona que la caña de azúcar se le puede utilizar para la elaboración de productos con valor agregado de diversas formas, entre los que podemos mencionar por ejemplo la melcocha y así estableciendo un nuevo nicho de mercado en la ciudad de Pasaje, priorizarlo al producto por ser nacional y artesanal a nivel local y regional, posicionándolo en el mercado competitivo a nivel de país con proyección a ser exportado. 
El mismo autor expresa que a pesar de contar con una considerable producción de caña de azúcar, en Ecuador, el mercado de confites está invadido por productos importados de una variedad de sabores, precios, tamaños, por lo cual los nuevos productos deben de tener un valor agregado que los diferencie del resto. Estos productos están dirigidos al público infantil, juvenil y adultos, debiendo escoger el target y mercado objetivo al que se dirige.

(Pachano, 2013) Como conclusiones de su investigación sobre comercio electrónico en el Ecuador: análisis de ventajas y desventajas de la compra y venta de productos a través del internet, expreso que genera una facilidad para que los consumidores puedan comprar productos desde sus hogares y a cualquier hora del día. En los países más desarrollados la compra y venta de productos a través del internet es muy común y cada vez de mayor volumen. En un país como el Ecuador existen muchas limitaciones y factores los cuales hacen que el comercio electrónico no tenga la misma acogida que en otros países más desarrollados.

El desarrollo del eCommerce (comercio electrónico) en Ecuador aún es incipiente y dentro de los países de América Latina que han movido compras online por \$ 75.000 millones, representa únicamente el 2\%. Lidera el ranking Brasil, que maneja el 60\% del comercio. Sin embargo, Ecuador tiene un potencial para este mercado: existe una creciente demanda y acceso a la tecnología. Así describió Marcos Pueyrredon, presidente del Instituto Latinoamericano de Comercio Electrónico. Jorge García, subdirector del INEC (Instituto Nacional de Estadística y Censos), explicó que en Ecuador existen 4’995.474 usuarios de redes sociales y que el promedio de uso por semana son 7,22 horas. Las provincias que encabezan en uso promedio de redes sociales son Guayas (7,35 horas semanales), Galápagos (con 7,35) y Pichincha (6,87). (El Universo, 2015)

En Ecuador según el INEC, se generaron transacciones en internet en 2014 por 540 millones de dólares, de los cuales 180 millones fueron compras por medio del sistema 4 x 4, que contaban con un valor promedio por compra de 74,45 USD y que según disposición de 2014 comenzaron a pagar adicional al transporte y servicio un valor fijo de 42 USD haciendo que esta opción sea poco atractiva y se haya reducido en más del 70\%. (Alcazar, 2015)

(Durán, 2014) Menciona que el 51\% de las compras virtuales se realiza en las tres ciudades más grandes del país: Quito, Guayaquil y Cuenca. Así lo dio a conocer en Instituto Ecuatoriano de Estadísticas y Censos (INEC) en el eCommerce Day, evento dedicado a la difusión del comercio electrónico, que se desarrolló en la ciudad de Guayaquil el 22 de julio pasado. Las 
prendas de vestir y el calzado son los productos más comprados por el ecuatoriano, lo que representa el $33 \%$ del total de adquisiciones.

La producción de dulces artesanales es una actividad tradicional propia de los diversos sectores de la provincia de Manabí, empezó como una tradición ancestral que se fue abriendo paso de generación en generación, pues era una actividad familiar que servía como un aporte económico en sus productores. La producción de dulces ha tenido un rápido incremento en años recientes en muchos países alrededor del mundo. Algunos de los factores que contribuyen al desarrollo de esta industria son: la mejora del método de manufactura y el desarrollo de equipos de procesamiento, ingredientes así como un mayor conocimiento en el uso de ellos, programas de publicidad y mercadeo de gran alcance, mantenimiento de la composición de los productos, calidad nutricional, productos agradables, florecimiento en los métodos de empaque y distribución a los consumidores. (Rivadeneira Astudillo, 2012)

La elaboración de alfeñique en Manabí es una actividad que en los últimos años ha incrementado, pues los niveles de demanda del producto son mayores. El alfeñique representa una alternativa de producción y rentabilidad económica en la microempresa Buena Fe, del recinto San Carlos, porque además de elaborar dicho producto, se obtienen otros dulces derivados de la caña de azúcar como panela, puntos y otros, además de licores como el aguardiente y guarapo.

El recinto San Carlos del cantón Jipijapa, es un sector dedicado a la producción netamente agrícola y pecuaria, dicho sector a su vez cuenta con pequeños sistemas de explotación en base a aprovechamiento de los cultivos característicos de la zona, como la caña de azúcar de la cual se extrae el jugo y luego de un proceso de cocción se obtiene la miel que es el componente principal para la elaboración de dulces artesanales y otros derivados de la misma. La microempresa Buena Fe, es una entidad dedicada a la producción y comercialización de productos derivados de la caña de azúcar, de manera principal los dulces como el alfeñique, punto, caramelos, panela, el guarapo y el aguardiente, producción que está destinada en mayor porcentaje al cantón Jipijapa.

El problema que dio origen a esta investigación es que la microempresa presenta deficiencias en los procesos de comercialización y ventas del producto, pues esta actividad la realizan de manera empírica, en gran parte es generado por el bajo nivel de preparación de los directivos del negocio quienes al desconocer diferentes métodos de comercialización no las aplican, esto 
ha dado como consecuencia bajos niveles de comercialización del producto, bajos ingresos para la microempresa y ha impedido su expansión; Se planteó como objetivo general, analizar el comercio electrónico en la comercialización de dulces artesanales de caña de azúcar por la microempresa buena fe y como objetivos específicos establecer la situación actual de la microempresa, en relación a la demanda de productos que elaboran; efectuar un diagnóstico sobre la producción y comercialización de dulces y derivados de la caña de azúcar por la microempresa e implementar una propuesta de innovación tecnológica que permita mejorar la comercialización de los dulces artesanales de cañar de azúcar.

\section{Materiales y métodos}

Para la realización de esta investigación se aplicó como métodos teóricos, el descriptivo, analítico, sintético y bibliográfico que ayudaron a establecer las bases teóricas de la investigación y a la vez ejecutar un análisis situacional de la comercialización de los productos que elabora la microempresa de dulces de fabricación artesanal, el método descriptivo se lo utilizó para realizar un estudio sobre uso de comercio electrónico y obtener bases sobre la comercialización de los productos que oferta la microempresa, el método analítico - sintético mediante el análisis de los resultados obtenidos en la recolección de datos del fenómeno de estudio para su posterior análisis y con ello llegar a realizar conclusiones y el bibliográfico basado en la búsqueda de información científica referente comercio electrónico y comercialización de dulces artesanales con el propósito de profundizar en las teorías para establecer o complementar nuevos conocimientos, en este caso el empleo de una estrategia de comercialización electrónica como acción a seguir para el aumento de oferta y venta de los productos elaborados por la microempresa; se utilizó la técnica de la encuesta para recopilar datos como las opiniones, necesidades y conocimientos revelados por los miembros de la microempresa Buena Fe en relación a la comercialización de dulces artesanales.

\section{Resultados}

Para la recolección de datos se aplicó una encuesta a los 17 miembros de la Microempresa Buena Fe, la cual se formuló a partir de la realización de un cuestionario, donde expresaron sus opiniones y necesidades en relación a la comercialización de los productos que ellos ofertan, de la cual se pudo obtener los siguientes resultados:

\begin{tabular}{|c|c|c|}
\hline Alternativas & Frecuencia & Porcentaje \\
\hline Alfeñique & 17 & $100 \%$ \\
\hline
\end{tabular}




\begin{tabular}{|c|c|c|}
\hline Panela & 0 & $0 \%$ \\
\hline Punto & 0 & $0 \%$ \\
\hline Licor & 0 & $0 \%$ \\
\hline Guarapo & 0 & $0 \%$ \\
\hline Total & $\mathbf{1 7}$ & $\mathbf{1 0 0 \%}$ \\
\hline
\end{tabular}

Tabla 1. Productos que elabora la Microempresa y demanda

Fuente: Miembros de la microempresa Buena Fe.

En relación al análisis de la pregunta para conocer qué tipo de productos elabora la microempresa Buena Fe, como se puede observar en la tabla \# 1 se procesan 5 productos diferentes derivados de la caña de azúcar los cuales pasan por diferentes fases de transformación hasta llegar a obtener el producto final tales como la panela, el punto, el licor, el guarapo (jugo de caña) y el alfeñique, siendo este último el que tiene mayor aceptación en el mercado, el 100\% de los encuestados mencionaron que los clientes prefieren este producto porque en relación a los otros, lo consumen niños, jóvenes y adultos, también porque se ofertan en 2 presentaciones, el alfeñique simple y el que va combinado con maní, hay dos opciones que van de acuerdo al gusto y preferencia del comprador, además porque es un producto natural que posee un agradable aroma y sabor.

\begin{tabular}{|c|c|c|}
\hline Alternativas & Frecuencia & Porcentaje \\
\hline Clientes directos & 17 & $100 \%$ \\
\hline Intermediarios & 0 & $0 \%$ \\
\hline Otros & 0 & $0 \%$ \\
\hline Total & $\mathbf{1 7}$ & $\mathbf{1 0 0 \%}$ \\
\hline
\end{tabular}

Tabla 2. Medios de comercialización del alfeñique Fuente: Miembros de la microempresa Buena Fe.

\begin{tabular}{|c|c|c|}
\hline Alternativas & Frecuencia & Porcentaje \\
\hline $\mathrm{Si}$ & 17 & $100 \%$ \\
\hline No & 0 & $0 \%$ \\
\hline Tal vez & 0 & $0 \%$ \\
\hline Total & $\mathbf{1 7}$ & $\mathbf{1 0 0 \%}$ \\
\hline
\end{tabular}

Tabla 3. Predisposición de ofertar sus productos mediante internet Fuente: Miembros de la microempresa Buena Fe.

En la tabla 2, se puede observar que referente a la pregunta direccionada para conocer los medios de comercialización que utiliza la micro empresa en la oferta del alfeñique, el 100\% de los consultados afirmó que lo realizan de manera directa a los clientes, pudiendo ser esta en las 
instalaciones del negocio, bajo pedido o mediante la venta en la feria libre que se realizan los fines de semana en la ciudad de Jipijapa y en menor proporción a pequeñas tiendas del mercado de la localidad mencionada, que no representa gran beneficio pues les cancelan poco por el producto; esto se da debido a que no cuentan con dinero suficiente, fuentes de financiamientos o alguna ayuda gubernamental para poder instalar locales o infraestructura física y ofrecer sus productos, también por el insuficiente conocimiento que tienen sobre métodos de comercialización sean estos físicos o virtuales que podrían implementar para llegar a otros mercados y captar nuevos clientes.

Como análisis de la presente interrogante, una vez que se les explico a los miembros de la microempresa Buena Fe sobre los beneficios de poder ofertar y vender sus productos mediante herramientas tecnológicos como el internet y que para poder realizar esta actividad no se necesita de ubicar una infraestructura física, se expone el criterio y decisión de sus integrantes en optar por utilizar sistemas informáticos de comercialización como es el internet, el 100\% de los encuestados expresó que si les gustaría, pues sería mecanismo que disminuye costos en los negocios comerciales y que es aplicado en la mayoría de las empresas a nivel mundial, mediante el análisis se determinó que la microempresa no utiliza herramientas tecnológicas, sin embargo tienen la predisposición de valerse de la tecnología para llegar a los clientes especificando las características de sus productos.

\begin{tabular}{|l|r|r|}
\hline \multicolumn{1}{|c|}{ Alternativas } & Frecuencia & Porcentaje \\
\hline Tiendas virtuales & 11 & $65 \%$ \\
\hline Correo electrónico & 0 & $0 \%$ \\
\hline Ventas telefónicas & 6 & $35 \%$ \\
\hline Otras & 0 & $0 \%$ \\
\hline Total & $\mathbf{1 7}$ & $\mathbf{1 0 0 \%}$ \\
\hline
\end{tabular}

Tabla 4. Elección de métodos de comercialización de sus productos con medios tecnológicos Fuente: Miembros de la microempresa Buena Fe.

En la ilustración se demuestra que los miembros de la Microempresa Buena Fe, en la interrogante acerca de la utilización de los medios tecnológicos en la comercialización de los dulces artesanales, un $65 \%$ afirmó que les gustaría utilizar tiendas virtuales, mientras el 35\% expreso que lo harían través del servicio de llamadas telefónicas, De acuerdo a estas características, el análisis muestra que se optaría por el empleo de tiendas virtuales, lo que les permitirá mostrar de manera diferente a la tradicional con el uso de la tecnología las 
características de los productos que producen y poder captar nuevos para llegar a los clientes, captarlos e incrementar sus ventas y tener desarrollo comercial.

\section{Discusión}

Una de las limitantes que tienen los microempresarios es la forma de cómo ellos comercializan sus productos pues por más que se esfuerzan es muy difícil llegar a posicionarse en el mercado, los altos costos que genera utilizar varias estrategias de marketing tradicionales, el entorno cambiante de los consumidores y al haber mucha competencia hace que en ocasiones los negocios no sean rentables y por ende sostenibles, por tal razón tenemos que buscar nuevas alternativas para dar a conocer y vender nuestros productos, una de ellas es utilizar herramientas tecnológicas como el comercio electrónico por medio de tiendas virtuales las cuales tienen la particularidad que podemos ofrecer productos y servicios las 24 horas del día, los 7 días de la semana y los 365 días del año sin que los clientes tengan que salir de sus hogares solo teniendo un computador o un dispositivo móvil, (Ehowenespanol.com, 2016) determina que el Internet reduce el tiempo de comercialización y disminuye los costos de comunicación, por lo que es una herramienta de comercio valorado por las empresas internacionales.

Sin embargo solo toma en cuenta a este tipo de negocios grandes, cuando debería también mencionar a las microempresas que de igual forma pueden optar por aplicar este tipo de herramientas tecnológicas considerando que son un pilar fundamental de desarrollo económico.

En la actualidad existen emprendedores que no tienen suficiente capital para poder instalar un negocio en forma física, pero que pueden optar por implementar sus negocios mediante el uso del comercio electrónico como es el caso de los propietarios de la microempresa Buena $\mathrm{Fe}$, que de acuerdo a la investigación realizada una de sus limitantes es que a pesar de producir un producto con una buena aceptación en el mercado tienen bajos niveles de comercialización, generado por ser una negocio pequeño y de bajos recursos pero con una gran predisposición de expandirse en el mercado.

(Bocanegra \& Vazquez, 2010) Afirmaron que el uso de las tecnologías permiten la reducción de costos de operación, menor tiempo en cada transacción con el cliente y en cada operación con el proveedor, concuerdo con los autores de este artículo aunque además es necesario mencionar que el beneficio es para ambas partes, en el caso de los empresarios porque no tienen que pagar mensualmente gastos corrientes de servicios básicos como agua, luz teléfono, 
remuneraciones de varios empleados y entre otros, lo cual le implicaría aumentar su margen de ganancias o poder bajar los precios de los productos que comercializa y a los clientes o usuarios porque pueden adquirir productos o servicios a precios más bajos y sin salir de su domicilio o el lugar donde se encuentren.

(Vivaelnetworking.com, 2015) Comenta que son cada vez más las compañías que incluyen los servicios web de comercio electrónico, así como aplicaciones para mantenerse en contacto directo y constante con el cliente, con el avance tecnológico constante podríamos decir que es un modelo de negocios del futuro, aunque también hay que tomar muy en cuenta los riesgos que se tienen al utilizar estas herramientas tecnológicas pues hay individuos que se dedican a falsear información al crear empresas fantasmas para estafar a clientes o como los denominados crackers que se encargan de vulnerar los sistemas informáticos.

\section{Conclusiones}

La microempresa Buena Fe es un negocio de tipo familiar que produce una gran variedad de productos derivados de la caña de azúcar, de manera principal los dulces como el alfeñique, punto, caramelos, panela, sus volúmenes de producción son considerables, los mismos que se han mantenido por la aceptación que tienen en el mercado.

Las principales debilidades que presenta la microempresa se centra en los procesos de comercialización, pues al desconocer y carecer de medios o herramientas adecuadas e innovadoras que permitan ampliar su mercado impiden que incrementen los volúmenes de ventas y en gran medida su desarrollo.

Los pequeños empresarios deben aprovechar las ventajas de aplicar herramientas tecnológicas como el comercio electrónico en la comercialización de sus productos a través de plataformas virtuales que permitan llegar hacia nuevos mercados y por lo tanto mejorar los niveles de ventas de los productos que ofertan.

\section{Referencias}

Alcazar, J. (18 de Agosto de 2015). Cifras, estadísticas y estado del e-commerce en Ecuador. Recuperado el 02 de Agosto de 2016, de www.elcomercio.com: 
http://www.elcomercio.com/tendencias/comercio-electronico-ecommerceemprendimiento-internet.html

Bocanegra, C., \& Vazquez, M. (2010). El uso de tecnología como ventaja competitiva en el micro y pequeño comercio minorista en Hermosillo, Sonora. Recuperado el 31 de Julio de 2016, de www.scielo.org.mx:

http://www.scielo.org.mx/scielo.php?script=sci_arttext\&pid=S018769612010000200008

Chillogalli, R. (2015). Diseño de un plan estratégico para la elaboración y comercialización de dulces derivados de la caña de azúcar en la ciudad de pasaje en el año 2015. Recuperado el 30 de Julio de 2016, de repositorio.utmachala.edu.ec: http://repositorio.utmachala.edu.ec/bitstream/48000/4779/1/TTUACE-2015-AECD00003.pdf

Durán, M. (05 de Agosto de 2014). El comercio electrónico se concentra en Quito, Guayaquil y Cuenca. Recuperado el 01 de Julio de 2016, de www.elcomercio.com: http://www.elcomercio.com/tendencias/comercio-electronico-ecommerceemprendimiento-internet.html

Ehowenespanol.com. (2016). El impacto del Internet y el comercio electrónico en negocios internacionales. Recuperado el 2 de Agosto de 2016, de www.ehowenespanol.com: http://www.ehowenespanol.com/impacto-del-internet-comercio-electronico-negociosinternacionales-lista_43591/

El Universo. (03 de Julio de 2015). Comercio electrónico requiere oferta interna. (http://www.eluniverso.com/noticias/2015/07/03/nota/4998307/comercio-electronicorequiere-oferta-interna ) Recuperado el 1 de Julio de 2016, de www.eluniverso.com: http://www.eluniverso.com/noticias/2015/07/03/nota/4998307/comercio-electronicorequiere-oferta-interna

Guerreo, M., \& Vera, J. (2016). “PROPUESTAS DE ESTRATEGIA DE MARKETING ELECTRÓNICO PARA LOS MICROEMPRESARIOS COMERCIALES DE GUAYAQUIL”. UNIVERSIDAD DE GUAYAQUIL, FACULTAD DE CIENCIAS ADMINISTRATIVAS, GUAYAQUIL. Obtenido de /repositorio.ug.edu.ec: http://repositorio.ug.edu.ec/bitstream/redug/10522/1/TESIS\%20LISTA\%201.pdf 
Landivar, D. (15 de Septiembre de 2012). Comercio electronico ¿ Canal de distrinbucion efectivo en el Ecuador? Recuperado el 2016 de Junio de 30, de www.fen.espol.edu.ec: http://www.fen.espol.edu.ec/landivardaniela_comercioelectronicocanaldedistribucione f ectivoenEcuador

Millan, R. (2016). marketingelectronico. Recuperado el 31 de Mayo de 2016, de www.ramonmillan.com: http://www.ramonmillan.com/tutoriales/marketingelectronico.php

Observatoriotic. (19 de Mayo de 2015). Comercio Electrónico en Ecuador. Recuperado el 31 de Mayo de 2016, de www.observatoriotic.mintel.gob.ec: http://www.observatoriotic.mintel.gob.ec/comercio-electronico-en-ecuador/

Pachano, J. (Mayo de 2013). Comercio electrónico en el Ecuador: análisis de ventajas y desventajas. Recuperado el 28 de jUNIO de 2016, de repositorio.usfq.edu.ec: http://repositorio.usfq.edu.ec/bitstream/23000/1834/1/106484.pdf

Posgradoenmarketing. (10 de Junio de 2014). ¿Qué es el Marketing en Comercio Electrónico? Recuperado el 31 de Mayo de 2016, de www.posgradoenmarketing.com: http://www.posgradoenmarketing.com/que-es-el-marketing-en-comercio-electronico

redempresariosvisa. (2016). ¿Qué es e-commerce o Comercio Electrónico? Recuperado el 31 de Junio de 2016, de www.redempresariosvisa.com: http://www.redempresariosvisa.com/Ecommerce/Article/que-es-e-commerce-ocomercio-electronico

Rivadeneira Astudillo, J. A. (2012). "Plan de negocio de una empresa que elabore y Comercialice los dulces artesanales de Rocafuerte Para la ciudad de quito”. Universidad De Las Américas, FACULTAD DE CIENCIAS ECONOMICAS Y ADMINISTRATIVAS.

Vivaelnetworking.com. (2015). La tecnología: Un medio de comercialización para el MLM. Recuperado el 1 de Agosto de 2016, de vivaelnetworking.com: http://vivaelnetworking.com/2015/02/la-tecnologia-un-medio-de-comercializacionpara-el-mlm/ 
Rev. SINAPSIS, Edición №12, Vol. 1, Diciembre 2018

ISSN 1390 - 9770 